\title{
Bloating and Distension in Irritable Bowel Syndrome: The Role of Gastrointestinal Transit
}

Anurag Agrawal, MBBS, MRCP ${ }^{1}$, Lesley A. Houghton, PhD, C Biol, FI Biol ${ }^{1}$, Brian Reilly, BSc ${ }^{1}$, Julie Morris, $\mathrm{MSc}^{2}$ and Peter J. Whorwell, MD, FRCP ${ }^{1}$

OBJECTIVES: Patients with irritable bowel syndrome and constipation (IBS-C) show more abdominal distension than do those with diarrhea. Because constipation is often associated with delayed gastrointestinal transit, this study aimed at establishing whether patients with delayed transit show more distension and bloating than do those with normal transit.

METHODS: $\quad$ Abdominal girth was recorded for $24 \mathrm{~h}$ using abdominal inductance plethysmography (AIP) in 30 IBS-C (Rome II criteria) patients (aged 18-68years; 27 women) and in 30 healthy volunteers (21-58 years of age; 26 women). Within 2 weeks of this recording, orocecal and colonic transits were assessed. Orocecal transit was determined from the increase in breath hydrogen after a standard meal, and colonic transit from the number of radio-opaque markers identified on a plain abdominal X-ray. Bloating severity was assessed hourly during AIP recording using a 0-5 scale.

RESULTS: $\quad$ IBS-C patients showed more bloating and distension than did healthy volunteers $(P<0.001)$. They also had prolonged colonic $(P<0.001)$ and orocecal $(P=0.05)$ transits than did healthy volunteers, such that 47 and $17 \%$, respectively, had colonic and orocecal transit times greater than the normal reference range. Those with delayed colonic $(P=0.025)$ and orocecal $(P=0.13)$ transits had greater distension but not bloating $(P=0.63$ and $P=0.48$, respectively) compared with those with normal transit. Moreover, distension directly correlated with orocecal $(r=0.40$; $P=0.03)$ and colonic $(r=0.51 ; P=0.004)$ transit times and inversely with stool consistency ( $r=-0.43 ; P=0.07)$.

CONCLUSIONS: IBS-C patients with delayed transit show greater abdominal distension than do those with normal transit. Therefore, drugs that accelerate transit may be expected to alleviate this troublesome problem.

Am J Gastroenterol advance online publication, 2 June 2009; doi:10.1038/ajg.2009.251

\section{INTRODUCTION}

Many patients with irritable bowel syndrome (IBS) and their physicians view the sensation of bloating as being synonymous with an actual increase in girth (i.e., distension). Indeed many investigators still use the terms interchangeably. However, a survey of patients with IBS has suggested that only approximately three-quarters of patients believe that their bloating is associated with actual abdominal distension, with this tending to be reported more often by patients with constipation than by those with diarrhea (1).
Using the validated ambulatory technique of abdominal inductance plethysmography (AIP) to objectively measure abdominal girth (2-4), we have shown recently that only approximately half of IBS patients with bloating show an actual increase in girth that is beyond that normally seen in healthy individuals (2). Furthermore, significantly more IBS-C patients (60\%) distended compared with patients with IBS and diarrhea (IBS-D) (40\%) (2). These findings, along with the observation that the severity of bloating only directly correlated strongly with the degree of abdominal distension in IBS-C (2), suggest that the pathophysiology is likely to be multifactorial and to differ between bowel habit subtypes.

${ }^{1}$ Neurogastroenterology Unit, Gastrointestinal Sciences, School of Translational Medicine, University of Manchester, Manchester, UK; ${ }^{2}$ Department of Medical Statistics, Wythenshawe Hospital, Manchester, UK. Correspondence: Lesley A. Houghton, PhD, C Biol, FI Biol, Neurogastroenterology Unit, Wythenshawe Hospital, Southmoor Road, Wythenshawe, Manchester, M23 9LT, UK. E mail: Lesley.Houghton@manchester.ac.uk

Received 14 November 2008; accepted 26 February 2009 
The pathophysiology of abdominal distension is still poorly understood. Studies have failed to show it to be related to excessive volumes of intra-abdominal gas $(5,6)$, and even in studies in which 10 times the normal amount of gas that is present in the gut has been infused into the intestine, this has been associated with only less than half of the mean increase in abdominal girth seen in IBS (i.e., $<2 \mathrm{~cm}$ ) (7), wherein changes can exceed $10 \mathrm{~cm}$ (2). However, rectal infusion of gas has been shown to be associated with a paradoxical relaxation of the internal oblique in patients with distension compared with an increase seen in healthy volunteers (8). This finding, together with the observation that basal electromyographic recordings of the anterior abdominal musculature in the absence of gas infusion show no differences between patients with and without a history of distension (9), might suggest an abnormality in an abdominal accommodation reflex rather than a weakness of the anterior abdominal wall. A contribution from motility is suggested by the observation that compared with both IBS-D patients and healthy controls, IBS-C patients retain and subsequently more slowly expel gas infused into the intestine (7). In addition, those with a history of bloating and distension $(\mathrm{B}+\mathrm{D})$ show delayed small-bowel transit (10), although not all studies have reproduced these findings (11). However, small-bowel manometry studies have been unable to show any differences during gas infusion between IBS patients and healthy controls (12). Similarly, in pilot studies, we have been unable to show any differences in small- and large-bowel phasic motor activity between occasions when IBS patients felt bloated and when they did not (13). Gastrointestinal transit can be viewed as a marker of motility, but this parameter has not been assessed earlier in relation to bloating or distension.

Therefore, the aim of this study was to use the validated objective technique of AIP to examine the relationship between objectively measured abdominal distension, the symptom of bloating, as well as orocecal and colonic transit in patients with IBS-C compared with those in healthy controls.

\section{METHODS}

\section{Patients}

Thirty patients aged 18-68 years (mean age 39 years, 27 women), who fulfilled the Rome II criteria for IBS-C (14) and specifically complained of a feeling of bloating that may be associated with a feeling of visible swelling of the abdomen, at least twice a week as a part of their symptom complex, were recruited from the outpatients department of the University Hospital of South Manchester NHS Foundation Trust. Specific questions asked included, "Do you suffer from the feeling of bloating or visible swelling of the tummy?" Patients who replied "yes" were then asked, "Do you suffer from this problem for at least 25\% of the time, ie at least two days a week?" All patients underwent appropriate investigations to exclude organic disease (15) and did not show any functional disorder of the upper gastrointestinal tract that was more prominent than their IBS. In addition, 30 age- and sex-matched healthy volunteers (aged 21-58 years, mean age 35 years, 26 women) were recruited for the study. Female participants were asked about their menstrual status to ascertain whether they were postmenopausal, had an earlier hysterectomy, or whether they had a regular menstrual cycle, and to ascertain the start date of their present cycle. All participants consumed below the recommended safe alcohol limit (women, <14 U/week; men, <20 U/week), and did not smoke or take medications that might affect gastrointestinal function at least $48 \mathrm{~h}$ before participating in the study. The study was approved by the South Manchester Local Research Ethics Committee, and all participants gave written informed consent.

\section{Protocol and Study Design}

All participants completed a 7-day diary to assess the severity of their IBS at the time of study. The presence and severity of abdominal bloating and pain/discomfort were scored at the end of the day using a $0-5$ scale $(0=$ none, $1=$ very mild, $2=$ mild, $3=$ moderate, $4=$ severe, and $5=$ very severe) and the time and consistency of any bowel movement $(1=$ very hard stool, $2=$ hard stool, $3=$ somewhat hard stool, $4=$ neither loose nor hard stool, $5=$ somewhat loose stool, $6=$ loose stool, and $7=$ watery stool), together with the presence of any straining at the time of stool passage, were noted (0-5 scale as above). Participants were classified as having a problem with straining if they scored 3 on at least two separate occasions.

After an overnight fast, patients attended the Neurogastroenterology Unit before 1000 hours and were fitted with the AIP device. Each patient returned the 7-day diary, completed the Hospital Anxiety and Depression (HAD) questionnaire (16), and was given a further paper diary in which to record, at "hourly intervals," the severity of any abdominal bloating and pain/discomfort (6-point Likert scale as above), the time and consistency of any bowel movement (1-7 scale as above), as well as the time and content of any oral intake (both solids and liquids). The patients were then allowed to leave the laboratory and were asked to maintain their usual daily activities until they returned for removal of the AIP device $24 \mathrm{~h}$ later, at which time they were asked to give an overall retrospective assessment of the severity of their bloating and pain/discomfort for the whole 24-h study period as none, mild, moderate, or severe (score $0-3$ ). Within 2 weeks, the patients underwent an assessment of their mouth to cecum transit and colonic transit.

\section{AIP}

The technique we used is described in detail elsewhere (2-4), but briefly, it works on the principle that a loop of wire forms an inductor, the inductance of which is dependent on the area enclosed by the loop. For the purposes of AIP, the wire is sewn into a band of elastic fabric ( $\approx 8.5 \mathrm{~cm}$ wide) in a zigzag manner to allow for expansion (Respitrace inductive sensor; Ambulatory Monitoring New York, NY), and is worn around the abdomen, similar to a belt. Attached to the wire is a small electronic circuit unit that incorporates an inductor in a resonant circuit, the output frequency of which varies with the area enclosed by the band, and a small battery-operated microprocessor data 
logger that records and scores the average frequency of the oscillator circuit for $30 \mathrm{~s}$ each minute. The data logger simultaneously records the patient's posture (standing, sitting, and lying down) through sealed mercury tilt switches (ASSEMtech Europe Ltd, Essex, UK) taped to the patients' chest and thigh. The cross-sectional area of the abdomen recorded by the equipment is then converted into a circumferential measurement, as described earlier (2-4).

\section{Colonic transit time}

Patients ingested three sets of radio-opaque markers (24 of each type) with $100 \mathrm{ml}$ of water at 0830 hours on 3 consecutive days. The three types of markers were cut from polyethylene tubing (Portex Limited, Nottingham, UK) as cylinders of identical mass with external diameter, internal diameter, and length of $4.5 \times 3 \times 1.3,3 \times 2 \times 3$, and $2 \times 1 \times 5 \mathrm{~mm}$, respectively. On the fourth day, after an overnight fast, the patients re-attended the hospital and a single abdominal X-ray was taken at 0830 hours. The numbers and location of markers on the film were used to calculate both colonic and segmental colonic transit time using the equation as suggested by Metcalf et al. (17). Immediately after the $\mathrm{X}$-ray, mouth to cecum transit was assessed.

\section{Mouth to cecum transit time}

The mouth to cecum transit time for the head of the meal was determined by a technique described earlier by Levitt (18). After rinsing the mouth with a $1 \%$ chlorhexidine mouthwash (Corsodyl P. 534 BNF (45), Glaxo SmithKline, Brentford, UK) baseline end expiratory breath samples were taken every $15 \mathrm{~min}$ for $1 \mathrm{~h}$. The patients then ingested a standard meal consisting of $30-\mathrm{g}$ dry flake potato reconstituted with $150-\mathrm{ml}$ water (Smash original, HL Foods Ltd, Spalding, Lincolnshire, UK; $107 \mathrm{kcal}$ ) and 120-g baked beans (HJ Heinz Co. Ltd, Hayes, Middlesex, UK; $83 \mathrm{kcal}$ ) within $5 \mathrm{~min}$, with $50 \mathrm{ml}$ of water, and immediately after consumption, rinsed their mouths again with Corsodyl mouthwash. Post-meal end expiratory breath samples were taken every $15 \mathrm{~min}$ for a maximum of $10 \mathrm{~h}$. Arrival of the head of the meal in the cecum was defined as the first time breath hydrogen increase by 10 p.p.m. above the baseline value and was sustained for three consecutive readings. Breath-hydrogen concentration was measured using a breath-hydrogen monitor (Gastrolyzer 2 Breath $\mathrm{H}_{2}$ Monitor, Bedfont Scientific Ltd., Rochester, Kent, UK) containing an electrochemical sensor, which was specific for hydrogen.

\section{Data analysis}

The subjective diary bloating scores for the hour at the start of the day and before retiring to bed on day 1 , and on waking on day 2 , were used to evaluate any diurnal variation in bloating. In addition, the mean bloating score from the beginning to the end of the day, referenced to the beginning of the day (i.e., mean bloating score from the second hour of the study to the end of day minus mean bloating score for the first hour of the study, representing an area under the curve (AUC)) were compared between patient groups.
As earlier studies have shown that there is no statistically significant difference between girth measurements taken in the standing and sitting positions (3), girth, whether in the sitting or standing position, was averaged over 60 -min epochs at the beginning of the day and before retiring to bed on day 1 , and immediately after waking on day 2 . In addition, the mean abdominal girth from the beginning to the end of the day, referenced to the beginning of the day (i.e., mean abdominal girth from the second hour of the study to the end of day 1 minus the mean girth for the first hour) was determined. As the data were distributed normally, absolute values and changes in abdominal girth and subjective bloating scores were compared between different times and patient groups using independent $t$-tests.

Similarly, orocecal and colonic transit times were compared between patient groups using independent sample $t$-tests.

In addition, each patient was classified as having clinically significant distension (i.e., B+D) or delayed orocecal, colonic, and segmental colonic transits if they showed values for these parameters above the 95th percentile limit for healthy volunteers. For example, patients with $\mathrm{B}+\mathrm{D}$ were defined as those with a mean abdominal girth from the beginning to the end of the day, referenced to the beginning of the day, which was above the 95th percentile limit for healthy volunteers, whereas those with values below the 95th percentile (i.e., within the normal reference range) were classified as having $B$ (bloating alone). Comparisons of transit times between patients with $\mathrm{B}+\mathrm{D}$ and $\mathrm{B}$, and those of distension between patients with delayed and normal transit times, were carried out using independent sample $t$-tests.

Correlations between mean values and changes in abdominal girth, as well as orocecal and colonic transits were performed using Pearson's test.

\section{RESULTS}

The demographic and baseline symptom profile of patients with IBS-C and that of healthy volunteers are summarized in Table 1. There were no differences in age, male-female ratio, body mass index, or baseline girth at the start of the day. In addition, there were similar numbers of women who were in the follicular (9 IBS-C vs. 10 healthy volunteers) or leuteal (8vs. 11) phases of their menstrual cycle, who were postmenopausal (7 vs. 5) or had earlier undergone hysterectomy (3 vs. 0). However, as expected, the patients complained of more bloating $(P<0.001)$ and pain/discomfort $(P<0.001)$, and opened their bowels less frequently $(P<0.001)$ with firmer stools $(P<0.001)$ than did healthy volunteers (Table 1 ). Moreover, significantly more IBS-C patients reported straining on bowel opening than did healthy volunteers, $(P<0.001)$ (Table 1), with nearly half of them reporting a bowel frequency of only 1-3 times a week and/or stool consistency score of between 1 and 2 on the Bristol Stool Scale. Patients with IBS-C were also significantly more anxious and depressed than were healthy volunteers $(P<0.001)$, although only 27 and $7 \%$ scored values that 
Table 1. Patient characteristics and baseline symptoms from 7-day diary

\begin{tabular}{|c|c|c|}
\hline & HV $(n=30)$ & IBS-C $(n=30)$ \\
\hline Age (years) & $35.30(31.27,39.33)$ & $39.33(34.81,43.85)$ \\
\hline Females:males & $26: 4$ & $27: 3$ \\
\hline $\begin{array}{l}\text { Body mass index } \\
\left(\mathrm{kg} / \mathrm{m}^{2}\right)\end{array}$ & $24.21(22.48,25.94)$ & $24.80(23.26,26.34)$ \\
\hline $\begin{array}{l}\text { Hospital anxiety } \\
\text { score }\end{array}$ & $4.25(3.22,5.28)$ & $7.60(6.30,8.90)^{*}$ \\
\hline $\begin{array}{l}\text { Hospital depression } \\
\text { score }\end{array}$ & $1.17(0.59,1.85)$ & $5.30(4.15,6.45)^{*}$ \\
\hline $\begin{array}{l}\text { Baseline girth at start } \\
(\mathrm{cm})\end{array}$ & $80.04(77.26,82.82)$ & $81.97(78.57,85.37)$ \\
\hline \multicolumn{3}{|c|}{ Baseline symptoms over 7 days } \\
\hline Bloating & $0.31(0.05,0.57)$ & $3.16(2.86,3.46)^{*}$ \\
\hline Pain/discomfort & $0.43(0.05,0.81)$ & $2.87(2.59,3.15)^{*}$ \\
\hline Stool consistency & $3.97(3.66,4.28)$ & $3.02(2.60,3.44)^{*}$ \\
\hline Stool frequency & $1.01(0.93,1.09)$ & $0.65(0.56,0.74)^{*}$ \\
\hline
\end{tabular}

No. (percentage) of patients with bowel frequency

$\begin{array}{lcc}>3 / \text { week } & 30(100 \%) & 17(56.6 \%)^{*} \\ 1 \text { to } 3 / \text { week } & 0 & 13(43.4 \%)^{*}\end{array}$

No. (percentage) of patients with stool consistency

$\begin{array}{lcc}\text { Score } 1 \text { or } 2 & 01(3.3 \%) & 14(46.7 \%)^{*} \\ \text { Score } 3 \text {, } 4 \text {, or } 5 & 28(93.3 \%) & 16(53.3 \%)^{\#} \\ \text { Score } 6 \text { or } 7 & 01(3.3 \%) & 0(0 \%)\end{array}$

No. reporting straining on bowel movement

$03(10 \%) \quad 23(76.7 \%)^{*}$

$\mathrm{HV}$, healthy volunteers; IBS-C, irritable bowel syndrome and constipation. Data expressed as mean and 95\% confidence interval. Baseline symptoms were averaged over the 7-day diary.

${ }^{*} P \leq 0.001$ compared with $\mathrm{HV}$; ${ }^{\#} P<0.01$ compared with $\mathrm{HV}$. were indicative of clinical anxiety and depression, respectively (i.e., a score $>10$ on the HAD questionnaire) (Table 1).

\section{Changes in abdominal girth (distension) and the symptom of bloating}

IBS-C patients reported significantly more bloating (i.e., the mean value from the beginning to the end of the day, referenced to the beginning of the day (AUC): 1.1; (95\% CI (95\% confidence interval): $0.7,1.5)$ vs. $(0(-0.1,0.1) ; P<0.001)$ and showed greater abdominal distension (AUC) $: 2.8 \mathrm{~cm}(2.0,3.6)$ vs. $(-0.4 \mathrm{~cm}(-1.0,0.2) ; P<0.001)$ than did healthy volunteers, with $62 \%$ showing distension beyond the 95 th percentile limit for healthy volunteers (i.e., $>2.30 \mathrm{~cm}$ ). As in earlier studies, both bloating and distension in IBS-C patients, but not in healthy volunteers, showed a diurnal increase, which returned to similar values overnight as those recorded at the beginning of the study $(P<0.05)$ (Table 2). Patients who scored the severity of their bloating as being greater than moderate (i.e., score of either 4 or $5 ; n=14$ ) for at least $2 \mathrm{~h}$ during the day showed greater abdominal distension than those who scored their bloating as being equal to or less than moderate during the day (i.e., score $\leq 3 ; n=16)(3.71 \mathrm{~cm}(2.75,4.61)$ vs. $1.93 \mathrm{~cm}(0.83$, 3.03); $P=0.02)$.

\section{Changes in orocecal and colonic transit}

Both orocecal (mean $(95 \%$ CI): 331.0 min $(295.8,366.2)$ vs. $287.0 \mathrm{~min}(259.0,315.0) ; P=0.05)$ and colonic $(51.8 \mathrm{~h}(46.2$, $57.4) \mathrm{h}$ vs. $34.8 \mathrm{~h}(28.9,40.7) \mathrm{h} ; P<0.001)$ transits were delayed in IBS-C patients compared with those in healthy volunteers. The delay in colonic transit in IBS-C patients seemed to be related to increased transit times throughout the colon, with the left $(23.1 \mathrm{~h}(20.1,26.1)$ vs. $14.0 \mathrm{~h}(10.1$, $17.9) ; P=0.001)$, right $(16.1 \mathrm{~h}(12.3,19.9)$ vs. $11.9 \mathrm{~h}(8.4$, $15.4) ; P=0.11)$, and rectosigmoid $(12.7 \mathrm{~h}(9.8,15.6)$ vs. $8.9 \mathrm{~h}$ $(6.8,11.0) ; P=0.04)$ regions showing increased transit times compared with those in healthy volunteers. Compared with the 95th percentile limits for healthy volunteers (orocecal transit, $412 \mathrm{~min}$; colonic transit, $61.1 \mathrm{~h}), 5$ (17\%) patients showed delayed orocecal transit and $14(47 \%)$ showed delayed colonic transit.

Table 2. Comparison of abdominal bloating and girth at the beginning and end of day 1 and the beginning of day 2 in IBS-C patients and healthy volunteers

\begin{tabular}{|c|c|c|c|c|}
\hline & \multicolumn{2}{|c|}{ Bloating Score } & \multicolumn{2}{|c|}{ Abdominal Girth $(\mathrm{cm})$} \\
\hline & HV & IBS-C & HV & IBS-C \\
\hline Beginning of day 1 & $0.2(0,0.4)$ & $1.6(1.2,2.0)^{*}$ & $80.0(77.2,82.8)$ & $82.0(78.5,85.5)$ \\
\hline Beginning of day 2 & $0(-0.1,0.1)$ & $2.0(1.7,2.3)^{*}$ & $78.6(75.6,81.6)$ & $80.9(76.9,84.9)$ \\
\hline
\end{tabular}



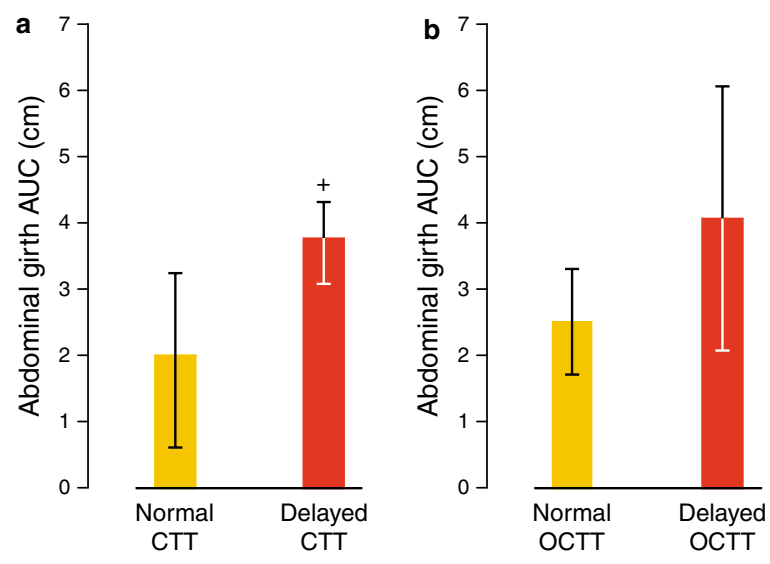

Figure 1. Effect of delayed colonic and orocecal transit on abdominal distension in irritable bowel syndrome and constipation (IBS-C). Comparison of abdominal distension in IBS-C patients with (a) normal and delayed colonic transit (CTT) and (b) normal and delayed orocecal transit (OCTT). ${ }^{+} P<0.05$ compared with those with normal CTT. AUC, area under the curve representing the mean abdominal girth from the second hour of the study to the end of day 1 minus the mean girth for the first hour.

\section{Relationship between abdominal distension and transit}

The 14 patients with delayed colonic transit showed greater distension than did the 16 patients with normal transit $(P=0.025)$. (Figure 1a). Those with delayed orocecal transit distended similarly to those with normal transit times $(P=0.13)$ (Figure 1b). However, although patients with delayed colonic transit reported firmer stools (Bristol Stool Score 1.4 (0.9, 1.9), mean $(95 \% \mathrm{CI})$ vs. $3.0(2.1,3.9) ; P=0.01)$, they did not report more bloating $(1.24(0.71,1.77)$ vs. $1.05(0.52,1.58) ; P=0.63)$, pain $(0.66(0.27,1.05)$ vs. $0.62(0.15,1.19) ; P=0.91)$, or fewer bowel movements per day $(0.91(0.53,1.29)$ vs. $0.93(0.53,1.33)$; $P=0.95)$ compared with those with normal transit. However, it is interesting that when patients were asked the next day to score their overall bloating for the previous day (retrospective score), those with delayed colonic transit reported more bloating $(2.28(1.06,3.50)$ and mean $(95 \% \mathrm{CI})$ vs. $1.63(1.17,2.09)$; $P=0.03)$ than did those with normal transit. Retrospective abdominal pain remained similar between groups (1.90 (1.40, $2.40)$ vs. $1.71(1.39,2.03) ; P=0.52)$.

Conversely, IBS-C patients who distended beyond the 95th percentile for healthy volunteers $(n=18)$, showed slower colonic $(P=0.008)$ and orocecal $(P=0.08)$ transits compared with those who distended within the normal reference range (Figure 2a and $\mathbf{b}$ ). Moreover, patients showing distension reported more bloating $(1.31(0.88,1.74)$ vs. $0.70(0.12,1.28)$; $P=0.11$ ) and firmer stools (Bristol Stool Score $1.7(0.8,2.6)$ vs. $2.9(2.0,3.8) ; P=0.06)$, but not pain $(0.57(0.15,0.99)$ vs. 0.69 $(0.25,1.13) ; P=0.70)$ or differences in bowel frequency $(1.10$ $(0.64,1.56)$ vs. $0.75(0.41,1.09)$ per day; $P=0.2)$ compared with those with bloating only. Again, the patients' retrospective score for bloating $(2.22(1.98,2.46)$ vs. $1.47(0.83,2.16)$; $P=0.02)$, but not pain $(1.76(1.36,2.16)$ vs. $1.81(1.37,2.25)$; $P=0.8)$, was greater in distenders than in non-distenders.
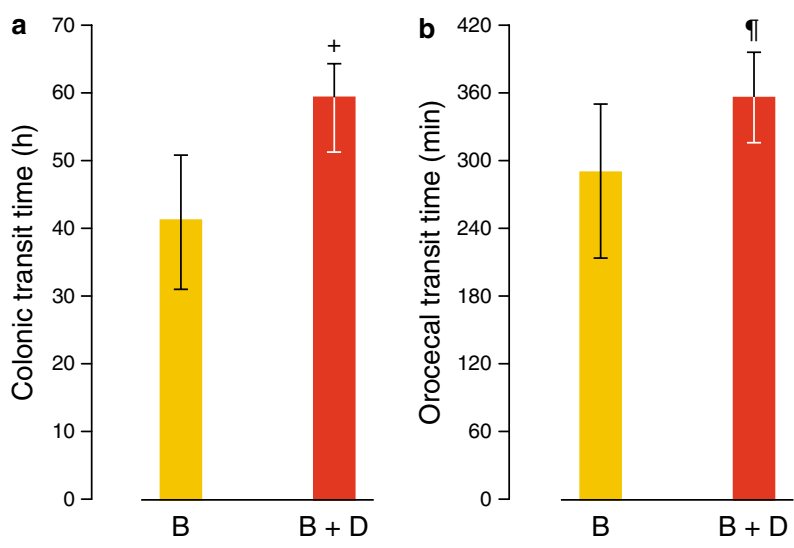

Figure 2. Effect of abdominal distension on colonic and orocecal transit in irritable bowel syndrome and constipation (IBS-C). Comparison of (a) colonic transit and (b) orocecal transit in IBS-C patients who distended $(B+D)$ compared with those who did not (B). ${ }^{+} P<0.05$ and $" P<0.1$ compared with non-distenders ( $\mathrm{B}$ alone). $\mathrm{B}$, bloating alone; $\mathrm{B}+\mathrm{D}$, bloating and distension.

\section{Correlations}

In patients with IBS-C, distension (i.e., mean from the beginning to the end of the day, referenced to the beginning of the day) directly correlated with abdominal bloating (again measured as the mean from the beginning to the end of the day, referenced to the beginning of the day) $(r=0.52, P=0.01)$ and orocecal $(r=0.40, P=0.03)$ and colonic $(r=0.51, P=0.004)$ transits. Moreover, distension also inversely correlated with the Bristol Stool Score $(r=-0.43 ; P=0.07)$ but not with stool frequency.

In contrast, bloating (i.e., mean from the beginning to the end of the day, referenced to the beginning of the day) did not correlate with either orocecal or colonic transit, or stool frequency or consistency.

Neither body mass index nor anxiety or depression correlated with any of the above parameters.

\section{DISCUSSION}

This is the first study to show a direct relationship between the physical sign of abdominal distension (i.e., increase in girth) and gastrointestinal transit time, in patients with IBS-C, particularly of the large bowel.

In accordance with our earlier studies (2), $~ 60 \%$ of IBS-C patients showed abdominal distension beyond the 95th percentile for healthy volunteers, with girth increasing as the day progressed, but improving or disappearing overnight. This was associated with a similar diurnal change in the symptom of bloating, such that there was a direct correlation between the severity of bloating and the amount of abdominal distension. IBS-C patients also showed slower colonic and orocecal transits compared with healthy volunteers, which is consistent with some (10) but not all (19) earlier studies in this patient group. However, this study has advanced earlier findings by showing that more IBS-C patients have delayed colonic (47\%) than 
orocecal (17\%) transit, probably explaining the reduced statistical significance observed for delayed orocecal transit compared with healthy volunteers.

The key finding in this study was that IBS-C patients who distend beyond the normal reference range showed both slower colonic and orocecal transits than did those with distension within the normal range. Indeed, the degree of distension directly correlated with both colonic and orocecal transit time. These data were reinforced by the observation that patients with transit times beyond the 95th percentile for healthy volunteers also had greater distension than did those with transit times within the normal range. Moreover, stool firmness, which is known to be inversely related to transit time (20), correlated with distension, whereas bowel frequency, which is not thought to relate well with transit time (20), did not. However, whether these findings reflect a non-specific effect of the constipation present in these IBS patients is unclear, and similar studies in patients with functional constipation are required to address this possibility. However, it is surprising that patients with delayed colonic transit reported a similar severity of bloating during the girth assessment period as did those with normal transit, although retrospectively they recalled more bloating. Although this may in part be related to the small number of patients in the two subgroups, it probably also reflects the subjective nature of the symptom of bloating and how it relates to the patients' interpretation of any physical distension, along with the fact that it is significantly associated with increased visceral sensitivity (21). However, it did not seem to be related to the psychopathology of the patients studied, as neither anxiety nor depression level correlated with bloating severity, distension, or indeed orocecal and colonic transits.

Increased intra-luminal content as a consequence of delayed transit being responsible for physically expanding the abdomen seems unlikely, given the fact that distension can reach up to $12 \mathrm{~cm}$ in some patients (2). This is supported by observations on ascites, in which relatively large volumes of fluid need to be retained before any noticeable change in girth occurs (22). It is also unlikely to be caused by voluntary protrusion of the abdomen (5), psychological factors (23), or lumbar lordosis (5). A more plausible explanation is that it is associated with some form of disturbance in an abdominal accommodation reflex, and this is supported by reports that, unlike healthy volunteers who show an increase in electromyographic activity to colonic gas infusion, some patients with a history of bloating fail to show tonic contraction of the abdominal wall and show a paradoxical relaxation of the internal oblique muscle (8). It is noteworthy that in this latter study, paradoxical relaxation of the internal oblique muscles was associated with abdominal distension, although it was not quite to the extent seen in this study. The particular viscerosomatic reflex involved is unknown, but it is of interest that in animal models of visceral sensation, increases in sensitivity are associated with changes in abdominal muscle contractility (24). Thus, it is possible to speculate that in a proportion of IBS-C patients in whom visceral hyposensitivity can occur, colonic stretching as a result of fecal loading might operate through the same reflex to relax, rather than contract, the abdominal musculature. Indeed, we have recently shown that hyposensitive IBS-C patients show the greatest degree of abdominal distension (21).

Finally, bloating and distension in patients with IBS has always been notoriously difficult to treat, but our finding that delayed transit is associated with abdominal distension suggests that drugs that accelerate transit could well play a role in the management of this problem. Indeed, it is noteworthy that we have recently shown that the probiotic, Bifidobacterium lactis DN-173 010, which significantly accelerates both orocecal and colonic transits in IBS patients with constipation, also reduces abdominal distension, as measured by the AIP technique (25), with the reduction in distension directly correlating with the improvement in transit. Moreover, neostigmine has been shown to accelerate gas transit and improve abdominal symptoms and distension in functional bowel patients with bloating (26), whereas the $5-\mathrm{HT}_{4}$ receptor agonist, tegaserod, has been shown to reduce bloating in patients with IBS-C (27-29), although no study to date has assessed the effects of this prokinetic on actual physical distension. These data support a real physiological link between delayed transit and abdominal distension in IBS patients with constipation, rather than delayed transit being just an epiphenomenon in patients who distend.

In conclusion, our data support a role for delayed transit in the physical manifestation of abdominal distension, and suggest that the use of agents that improve transit might be an appropriate approach to treat this often troublesome problem.

\section{CONFLICT OF INTEREST}

Guarantor of the article: Lesley A. Houghton, PhD, C Biol, FI Biol.

Specific author contributions: Study design and completion of practical aspects of study, majority of the writing, study analysis and statistics, and majority of the patient referrals: Anurag Agrawal; study conception and design, study interpretation and analysis, and principal writing: Lesley A. Houghton; design of electronics and computer programs for abdominal inductance plethysmography (AIP) device: Brian Reilly; study analysis and statistics and manuscript review: Julie Morris; manuscript review and writing and patient referral: Peter J. Whorwell.

Financial support: This study was kindly funded in part by an educational grant from Novartis Pharmaceuticals, Basel, Switzerland.

Potential competing interests: Lesley A. Houghton has served as a speaker, a consultant, and/or an advisory board member for Novartis, Pfizer, Solvay Pharmaceuticals, GlaxoSmithKline, Clasado, and the department has received research funding from Novartis, Pfizer, Solvay Pharmaceuticals, GlaxoSmithKline, and Danone Research. Lesley A. Houghton is an employee of the University of Manchester. 
Peter J. Whorwell has served as a speaker, consultant, and as an advisory board member for Novartis Pharmaceuticals, GlaxoSmithKline, Solvay Pharmaceuticals, Rotta Research, Procter and Gamble, Danone Research, Astella Pharma, and Tillots Pharma, and the department has received research funding from Novartis Pharmaceuticals, GlaxoSmithKline, Solvay Pharmaceuticals, Rotta Research, Procter and Gamble, and Danone Research. Peter J. Whorwell is an employee of the University of Manchester and University Hospital of South Manchester NHS Foundation Trust. All other authors declare no conflict of interest.

\section{Study Highlights}

\section{WHAT IS CURRENT KNOWLEDGE}

Patients with irritable bowel syndrome and constipation (IBS-C) show more abdominal distension (i.e., increase in girth) than do those with diarrhea alone.

Constipation is often associated with delayed transit.

\section{WHAT IS NEW HERE}

This is the first study to show that abdominal distension directly correlates with orocecal and colonic transits, and inversely with stool consistency.

IBS-C patients with delayed transit show greater distension than do those with normal transit.

These results, together with our earlier findings that accelerating transit improves distension, suggest that delayed transit is a real physiological cause for the distension in these patients.

Therefore, accelerating transit or relieving constipation might well be a useful approach to reducing distension, which, until now, has proved to be notoriously difficult to treat.

\section{REFERENCES}

1. Chang L, Lee O-Y, Naliboff B et al. Sensation of bloating and visible abdominal distension in patients with irritable bowel syndrome. Am J Gastroenterol 2001;96:3341-7.

2. Houghton LA, Lea R, Agrawal A et al. Relationship of abdominal bloating to distension in irritable bowel syndrome and the effect of bowel habit. Gastroenterology 2006;131:1003-10.

3. Lewis MJ, Reilly B, Houghton LA et al. Ambulatory abdominal inductance plethysmography: towards objective assessment of abdominal distension in irritable bowel syndrome. Gut 2000;48:216-20.

4. Reilly BP, Bolton M, Lewis MJ et al. A device for 24 hour ambulatory monitoring of abdominal girth using inductive plethysmography. Physiol Meas 2002;23:661-70.

5. Maxton DG, Martin DF, Whorwell PJ et al. Abdominal distension in female patients with irritable bowel syndrome: exploration of possible mechanisms. Gut 1991;32:662-4.

6. Lasser RB, Bond JH, Levitt MD. Role of intestinal gas in functional abdominal pain. N Engl J Med 1975;293:524-6.
7. Serra J, Azpiroz F, Malagelada JR. Impaired transit and tolerance of intestinal gas in the irritable bowel syndrome. Gut 2001;48:14-9.

8. Tremolaterra F, Villoria A, Azpiroz F et al. Impaired viscerosomatic reflexes and abdominal-wall dystony associated with bloating. Gastroenterology 2006;130:1062-8.

9. McManis PG, Newall D, Talley NJ. Abdominal wall muscle activity in irritable bowel syndrome with bloating. Am J Gastroenterol 2001; 96:1139-42.

10. Cann PA, Read NW, Brown C et al. Irritable bowel syndrome: relationship of disorders in the transit of a single solid meal to symptom patterns. Gut 1983;24:405-11.

11. Hebden JM, Blackshaw E, D'Amato $M$ et al. Abnormalities of GI transit in bloated irritable bowel syndrome: effect of bran on transit and symptoms. Am J Gastroenterol 2002;97:2315-20.

12. Galati JS, McKee DP, Quigley EM. Response to intraluminal gas in irritable bowel syndrome. Motility versus perception. Dig Dis Sci 1995;40:1381-7.

13. Lewis MJV, Houghton LA, Whorwell PJ. Changes in small and large bowel phasic activity do not explain the the increased perception of distension in patients with IBS. Gut 2001;49 (Suppl III): 3088.

14. Thompson WG, Longstreth GF, Drossman DA et al. Functional bowel disorders and functional abdominal pain. Gut 1999;45 (Suppl 2): 1143-7.

15. Drossman DA, Camilleri M, Mayer EA et al. AGA technical review on irritable bowel syndrome. Gastroenterology 2002;123:2108-31.

16. Zigmond AS, Snaith RP. The hospital anxiety and depression scale. Acta Psychiatr Scand 1983;67:361-70.

17. Metcalf AM, Phillips SF, Zinsmeister AR et al. Simplified assessment of segmental colonic transit. Gastroenterology 1987;92:40-7.

18. Levitt MD. Production and excretion of hydrogen gas in man. N Engl J Med 1969;28:122-7.

19. Horikawa Y, Meino H, Inoue M et al. Gastrointestinal motility in patients with irritable bowel syndrome studied by using radiopaque markers. Scand J Gastroenterol 1999;34:1190-5.

20. O’Donnell LJD, Virjee J, Heaton KW. Detection of pseudodiarrhoea by simple clinical assessment of intestinal transit rate. Br Med J 1990;300: 439-40.

21. Agrawal A, Houghton LA, Lea R et al. Bloating and distension in irritable bowel syndrome: the role of visceral sensation. Gastroenterology 2008;134:1882-9.

22. Cattau EI, Benjamin SB, Knuff TE et al. The accuracy of the physical exam in the diagnosis of suspected ascites. JAMA 1982;247:1164.

23. Houghton LA, Whorwell PJ. Towards a better understanding of abdominal bloating and distension in irritable bowel syndrome. Neurogastroenterol Motil 2005;17:500-11.

24. Ness TJ, Gebhart GF. Colorectal distension as a noxious visceral stimulus: physiologic and pharmacologic characterization of pseudoaffective reflexes in the rat. Brain Res 1988;450:153-16.

25. Agrawal A, Houghton LA, Morris J et al. Clinical trial: the effects of a fermented milk product containing Bifidobacterium lactis DN 173010 on abdominal distension and gastrointestinal transit in irritable bowel syndrome with constipation. Aliment Pharmacol Ther 2008;29:104-14.

26. Caldarella MP, Serra J, Azpiroz F et al. Prokinetic effects in patients with intestinal gas retention. Gastroenterology 2002;122:1748-55.

27. Muller-Lissner SA, Fumagalli I, Bardhan KD et al. Tegaserod, a 5-HT(4) receptor partial agonist, relieves symptoms in irritable bowel syndrome patients with abdominal pain, bloating and constipation. Aliment Pharmacol Ther 2001;15:1655-66.

28. Novick J, Miner P, Krause R et al. A randomized, double-blind, placebocontrolled trial of tegaserod in female patients suffering from irritable bowel syndrome with constipation. Aliment Pharmacol Ther 2002;16: 1877-88.

29. Whorwell PJ, Ruegg P, Earnest D et al. Tegaserod significantly improves bloating in female irritable bowel syndrome patients with constipation. Gastroenterology 2004;126:A643. 